\title{
On the Crustal Matter of Magnetars
}

\author{
Nandini Nag and Somenath Chakrabarty ${ }^{\dagger}$ \\ Department of Physics, Visva-Bharati, \\ Santiniketan 731 235, West Bengal, India \\ ${ }^{\ddagger}$ E-mail:somenath.chakrabarty@visva-bharati.ac.in
}

\begin{abstract}
We have investigated some of the properties of dense sub-nuclear matter at the crustal region (both the outer crust and the inner crust region) of a magnetar. The relativistic version of ThomasFermi (TF) model is used in presence of strong quantizing magnetic field for the outer crust matter. The compressed matter in the outer crust, which is a crystal of metallic iron, is replaced by a regular array of spherically symmetric Wigner-Seitz (WS) cells. In the inner crust region, a mixture of iron and heavier neutron rich nuclei along with electrons and free neutrons has been considered. Conventional Harrison-Wheeler (HW) and Bethe-Baym-Pethick (BBP) equation of states are used for the nuclear mass formula. A lot of significant changes in the characteristic properties of dense crustal matter, both at the outer crust and the inner crust, have been observed.
\end{abstract}

PACS numbers: 97.60.Jd, 97.60.-s, 75.25.+z 


\section{INTRODUCTION}

Magnetars are the most exotic stellar objects, believed to be strongly magnetized young neutron stars. The surface magnetic field for such objects are observed to be $\geq 10^{15} \mathrm{G}$ $[1,2$, 3, 4]. Then it is quite possible that the field at the interior, even at the inner crust region may be stronger than the surface value (predicted theoretically by scalar Virial theorem). If the internal field strength is happened to be so high, then most of the physical and chemical properties of dense stellar matter of the magnetars must change significantly from the conventional picture [5, 6, 7]. A lot of investigations have already been done on the effect of strong quantizing magnetic field on various physical properties of dense stellar matter inside neutron stars as well as quark matter inside quark stars or hybrid stars, including the effect on quark-hadron phase transition at the core region of a compact neutron star [8]. The effect of such strong magnetic field on various elementary processes inside neutron stars and quark stars or hybrid stars have also been studied. The effect of strong quantizing magnetic field on the $\beta$-equilibration among the constituents have also been investigated

[9]. It has also been shown that strong quantizing magnetic field acts like a catalyst to generate fermion mass dynamically, i.e. chiral symmetry breaking occurs in presence of strong quantizing magnetic field [10, 11, 12].

In this article we have presented our investigation on the effect of strong magnetic field on the crustal matter of magnetars. The work is divided into two parts: in the first part, based on one of our very recent work [13], we have investigated the effect of strong quantizing magnetic field on the outer crust matter and in the second part, we study the properties of compact sub-nuclear matter at the inner crust region in presence of such strong quantizing magnetic field.

The paper is organized in the following manner: In section 2, the effect of strong quantizing magnetic field on the outer crust matter of magnetars is discussed, in section 3, we have presented with detailed numerical computation on the effect of strong magnetic field on the inner crust matter, which is assumed to be a mixture of iron, some heavier neutron rich nuclei, electrons and free neutrons. The presence of free neutrons are considered beyond neutron drip density. In this section, we have re-investigated the properties of inner crust matter of a typical neutron star in presence of strong quantizing magnetic field. In the inner crust region, for metallic iron and more heavier neutron rich nuclei, we have considered the 
conventional HW and BBP equation of states [14, 15, 16]. Finally, in the last section, we have given the conclusions and discussed the importance and future prospects of the present work.

\section{OUTER CRUST MATTER}

In a recent work we have developed an exact formalism for the relativistic version of TF model in presence of strong quantizing magnetic field [13]. This formalism is used within the limitation of TF model [17], to obtain the equation of state state for crustal matter of a typical magnetar, which is mainly a dense crystal of metallic iron in the sub-nuclear density region. In this model, the compressed iron atoms / ions are replaced by the spherically symmetric WS cells, with positively charged nuclei at the centre, surrounded by a nonuniform cloud of electron gas. In this model it has been assumed that in a WS cell, the atomic number which is the number of protons within the nuclei is $Z$ and the mass number is $A$ (number of protons and neutrons). To make each cell electrically charge neutral, $Z$ must also be the number of electrons inside the WS cells.

In this model, the modified form of Poisson's equation is given by [13]

$$
\frac{d^{2} \phi}{d x^{2}}=\sum_{\nu=0}^{\nu_{\max }}\left(2-\delta_{\nu 0}\right)\left(\phi^{2}(x)-\phi_{0}^{2} x^{2}\right)^{1 / 2}
$$

where $\phi(x)$ is the modified form of electrostatic potential related to the original Coulomb potential $V(x)$ by the relation

$$
\phi(x)=\frac{\mu x}{Z e^{2}}\left[\mu_{e}+e V(x)\right]
$$

with $\mu_{e}$, the electron chemical potential, assumed to be constant throughout the cell (this is the so called Thomas-Fermi condition), the dimensionless scaled radial coordinate $x$ is related to the actual radial coordinate $r$ of WS cells by the relation: $x=r / \mu$, with

$$
\mu=\left(\frac{\pi}{2 e^{3} B}\right)^{1 / 2}
$$

$e=|e|$, the magnitude of electron charge, $B$ is the constant magnetic field, assumed to be along $z$-direction (we have chosen the gauge $A^{\mu} \equiv(0,0, x B, 0)$ ),

$$
\phi_{0}=\frac{m_{\nu} \mu}{Z e^{2}}
$$


with $m_{\nu}=\left(m_{e}^{2}+2 \nu e B\right)^{1 / 2}, m_{e}=0.5 \mathrm{MeV}$, the electron rest mass and $\nu=0,1,2, \ldots \ldots, \nu_{\max }$, is the Landau quantum number for the electrons, $\nu_{\max }$ is the upper limit of Landau quantum number (the upper limit of the Landau quantum number $\nu_{\max }$ is finite at zero temperature, otherwise $\left.\nu_{\max }=\infty\right)$. Finally, the factor $\left(2-\delta_{\nu 0}\right)$ indicates that the zeroth Landau level is singly degenerate, whereas, all other states are doubly degenerate. In this article we have assumed that the electron gas in the dense crustal matter of metallic iron crystal is strongly degenerate and is considered to be at zero temperature. Then it is quite obvious from the non-negative value of electron Fermi momentum, that the upper limit of Landau quantum number $\nu_{\max }$ is given by

$$
\nu_{\max }=\frac{\mu_{e}^{2}-m_{e}^{2}}{2 e B}
$$

To obtain numerical solution for $\phi(x)$ for a given magnetic field and a particular set of $Z$ and $A$, we further assume that instead of a point object, the nucleus at the centre of a WS cell, has a finite dimension and is assumed to be spherical in nature, so that the corresponding radius $r_{n}=r_{0} A^{1 / 3}$, with $r_{0}=1.12 \mathrm{fm}$. Such a choice also removes the singularity problem of TF equation at the origin [19]. Therefore it is not necessary to follow the prescription given by Feynman, Metropolis and Teller to obtain the numerical solution for TF equation [20]. Again from the physics point of view, the potential must satisfy the boundary conditions, given by

$$
r V(r)=Z e \quad \text { for } \quad r \rightarrow r_{n}, \quad \text { and } \quad \frac{d V}{d r}=0 \quad \text { for } \quad r \rightarrow r_{s}
$$

where $r_{s}$ is the radius of the WS cell. Then by simple algebraic manipulation it is easy to show that $\phi(x)$, the modified form of Coulomb potential satisfies the boundary conditions

$$
\left.\phi(x)\right|_{x=x_{n}}=1 \quad \text { and }\left.\quad \frac{d \phi}{d x}\right|_{x=x_{s}}=\left.\frac{\phi(x)}{x}\right|_{x=x_{s}},
$$

where $x_{n}=r_{n} / \mu$, the scaled nuclear radius and $x_{s}=r_{s} / \mu$, the corresponding scaled radius of the WS cell. Both these quantities are dimensionless.

Further, the right hand side of the Poisson's equation (eqn.(1)) must be real. Which requires $\phi_{0} x \leq|\phi(x)|$. Hence we get an additional condition, to be satisfied by the upper limit of Landau quantum number, and is given by

$$
\nu_{\max }(x) \leq\left(\frac{e^{6} Z^{2}}{\pi x^{2}} \phi(x)^{2}-\frac{m_{e}^{2}}{2 e B}\right),
$$

From the definition of Landau quantum number, the above inequality must necessarily be $\geq 0$. The above equation also shows that the upper limit of Landau quantum number 
depends on the position ( $x$ or $r$ coordinates) of the electron within the WS cell, with which it is associated.

Since electron distribution is non-uniform within each cell, the Fermi momentum of a particular electron must depend on its positional coordinate in the cell. Then it is expected that the variation of $p_{F}(r)$ will be such that the electron chemical potential, given by

$$
\mu_{e}=\left(p_{F}(r)^{2}+m_{e}^{2}+2 \nu(r) e B\right)^{1 / 2}
$$

remain constant throughout the cell with proper space dependent Landau quantum number $\nu$.

Satisfying all these conditions, of which some of them are particularly necessary for this model, we have solved the Poisson's equation numerically within the range of $r$ from nuclear surface to the WS cell boundary, for $B=B_{c}^{(e)}, 10 \times B_{c}^{(e)}, 10^{2} \times B_{c}^{(e)}$ and $10^{3} \times B_{c}^{(e)}$, where $B_{c}^{(e)}$ is the quantum critical limit for the magnetic field at and above which the Landau levels are populated for the relativistic electrons, and is given by $e B_{c}^{(e)}=m_{e}^{2}$ with the choice of unit $\hbar=c=1$. The magnetic field beyond this limit is called the quantizing magnetic field and the quantum mechanical effect plays an important role in this domain. Within the range $x_{n} \leq x \leq x_{s}$, the numerical solution for $\phi(x)$ can be fitted exactly by a straight line for a particular magnetic field strength $B$ and can be expressed as $\phi(x)=a x+b$. Obviously, the parameters $a$ and $b$ are functions of magnetic field strength. In TABLE-I we have shown the variation of the parameters $a$ and $b$ with magnetic field strength.

TABLE-I

\begin{tabular}{|l|c|c|c|c|c|c|r|}
\hline$B / B_{c}^{(e)}$ & $a$ & $b$ & $x_{s}\left(\mathrm{MeV}^{-1}\right)$ & $\alpha$ & $\beta$ & $p$ & $q$ \\
\hline $10^{0}$ & -2.48 & 1.0002 & 30.67 & 0.0113 & 2.694 & 0.04 & 0.574 \\
$10^{1}$ & -2.54 & 1.0001 & 11.78 & 0.0789 & 2.602 & 0.25 & 0.591 \\
$10^{2}$ & -2.69 & 1.0001 & 4.58 & 0.7157 & 2.504 & 0.39 & 0.743 \\
$10^{3}$ & -3.08 & 1.0002 & 1.78 & 5.0198 & 1.948 & 0.52 & 0.842 \\
\hline
\end{tabular}

(Variation of the parameter $a$ and $b$ for $\phi(x)=a x+b$ and scaled surface radius $x_{s}$ for WS cells, parameters $\alpha$ and $\beta$ for $E_{K E}=\alpha x^{\beta}$ and the parameters $p$ and $q$ for $E_{e e}^{\mathrm{ex}}=p x^{q}$ with magnetic field strength)

Because of some numerical in-accuracy, the values of the parameter $b$, give the intersections of the straight lines with $y$-axis, are not exactly one. Instead of fixing its exact value 
which is equal to one for all $B$, and fitting the straight lines with $a$ as unknown parameter, we have used our numerical code for general purpose. However, for all the magnetic field values, the parameter $b$ is very close to one. The numerical values for the other parameter $a$ is always negative and its magnitude increases with the increase in magnetic field $B$. Which actually means that the radius or the volume of a particular WS cell decreases with the increase in magnetic field strength. In TABLE-I we have also shown the explicit variation of $x_{s}$, the scaled surface radius of the WS cells, for various magnetic field strength. While solving the Poisson's equation numerically for a given magnetic field strength $B$, the instruction is given in our numerical code to terminate if the surface condition, given by eqn.(7) is satisfied and hence we obtain $x_{s}\left(\right.$ also $\left.r_{s}=x_{s} \mu\right)$ as a function of magnetic field strength. In fig.(1) we have shown the variation of $x_{s}$ and also the corresponding $r_{s}$ with the strength of magnetic field. This figure clearly shows that the WS cells become more compressed if the magnetic field becomes stronger. This is in some sense analogous to what is called the magnetostriction in classical magneto-statics. These two curves can also be fitted by the power law functions, given by

$$
x_{s}=29.43 \times\left(\frac{B}{B_{c}^{(e)}}\right)^{-0.41} \mathrm{MeV}^{-1} \text { and } r_{s}=1.704 \times\left(\frac{B}{B_{c}^{(e)}}\right)^{-0.91} \AA
$$

Knowing the scaled Coulomb potential $\phi(x)$ at various $x$ points (in the range $x_{n}$ to $x_{s}$ ) within the WS cell for a given magnetic field strength, we have evaluated numerically $\nu_{\max }(x)$ at every $x$ points within the cell. The variations of $\nu_{\max }(x)$ with $x$ for four different magnetic field strengths are shown in fig.(2). Although $\nu_{\max }(x)$ must be a set of discrete numbers, for the sake of illustration, we have plotted it as a continuous variable. In this figure, curves $a$ and $b$ are for $B=B_{c}^{(e)}$ and $10 \times B_{c}^{(e)}$ respectively. For these two curves, the variables $\nu_{\max }(x)$ is plotted along the left side $y$-axis and $x$ (this is actually $x \mu$ in $\mathrm{Mev}^{-1}$ ) along $x$-axis at the bottom. Similarly for $B=50 \times B_{c}^{(e)}$ and $100 \times B_{c}^{(e)}$, the variations are shown by the curves $\alpha$ and $\beta$ respectively. In this case, $\nu_{\max }(x)$ is plotted along the right side $y$-axis and $x$ is plotted along upper $x$-axis. From this figure, it is possible to make a number of conclusions: (i) For larger $B$ values, $x_{s}$ are smaller. (ii) For smaller $B, \nu_{\max }(x)$ starts with quite large value near the nuclear surface, e.g., $=124$ and $=15$ as shown in curves $a$ and $b$ respectively. Whereas, for curve $\alpha$ it starts with $\nu_{\max }=2$ and for $\beta$, the starting value is $\nu_{\max }=1$. (iii) The discrete nature of $\nu_{\max }$ is obvious from the high magnetic field curves $\alpha$ and $\beta$. (iv) Finally, for all the field values, the upper limit $\nu_{\max }(x)$ becomes exactly zero at the surface 
of the WS cells. In other wards, we can say that all the electrons near the WS cell surface are strongly polarized and the spins are anti-parallel to the direction of magnetic field. This is, of course, a purely relativistic effect. The possibility of fully polarized scenario can easily be obtained from the analytical solution of Dirac equation for electrons in presence of strong quantizing magnetic field. The eigen functions will not be simple spinor solutions, whereas the energy eigen value will be $E_{\nu}=\left(p_{z}^{2}+2 \nu e B+m_{e}^{2}\right)^{1 / 2}$, with $2 \nu=n+1+m_{s}$, where $n=0,1,2, .$. is the Landau principal quantum number and $m_{s}= \pm 1$, the eigen values for the spin operator $\sigma_{z}[13,21]$. Hence, for $\nu=\nu_{\max }=0$, the only possible choice is the combination $n=0$ and $m_{s}=-1$. Which actually means that in the zeroth Landau level the spins of all the electrons are in the direction opposite (this is due to negative charge carried by the electrons) to the external magnetic field. We have further noticed that beyond the field value $100 \times B_{c}^{(e)}$ (which is $>10^{15} \mathrm{G}$ ), the upper limit $\nu_{\max }(x)$ becomes identically zero not only at the surface region of WS cells, but at all the points inside the cell.

To obtain the density distribution of electrons within the WS cells, let us consider the expression for electron number density, given by

$$
n_{e}(x)=\frac{e B}{2 \pi^{2}} \sum_{\nu=0}^{\nu_{\max }(x)}\left(2-\delta_{\nu 0}\right) p_{F}(x)
$$

where the electron Fermi momentum $p_{F}(x)$ can be obtained from the TF condition and is given by

$$
p_{F}(x)=\left\{Z^{2} e^{4}\left(\frac{\phi(x)}{\mu x}\right)^{2}-m_{\nu}^{2}\right\}^{1 / 2}
$$

Since the electron density is larger near the nuclear surface, i.e., at the central region of the WS cells compared to the values near their boundary regions, the effect of electron density dominates over the influence of magnetic field strength at the central region, whereas near the WS surface, since the density is low enough, it will be of completely opposite picture; the magnetic field will play the significant role. This will make the upper limit $\nu_{\text {max }}$ large enough (except for $B \geq 10^{15} \mathrm{G}$ ) near the nuclear surface and quite small or identically zero near WS cell boundary. The results are also quite obvious because the electron Fermi momentum is minimum at the nuclear surface and maximum near the the WS cell boundary. Later we shall see that the electron kinetic energy part will also behave like Fermi momentum. Therefore. the quantum mechanical effect of low and moderate values for quantizing magnetic field will be more significant near the surface region of WS cells. Near the core region, the effect is 
almost classical. This is particularly valid for the curves $a$ and $b$ of fig.(2), because for these curves $\nu_{\max }(x)$ started with quite large values.

It is also obvious from eqn(11), that the electron Fermi momentum is a function of the positional coordinate of the associated electron. Using the numerically fitted form of $\phi(x)$ we obtain $p_{F}(x)$ for a given magnetic field strength and the corresponding $n_{e}(x)$ for the same magnetic field. In fig.(3) we have plotted the variation of electron density within WS cells for four different magnetic field strengths. In this figure, the curves indicated by the numbers $0,1,2$ and 3 are for $B=B_{c}^{(e)}, B=10 \times B_{c}^{(e)}, B=10^{2} \times B_{c}^{(e)}$ and $B=10^{3} \times B_{c}^{(e)}$ respectively. It is quite obvious from these curves that the electron density increases with the increase in magnetic field strength. Further, for all the values of $B$, electron density is maximum at the centre and minimum at the surface.

Let us now evaluate the variation of (i) electron kinetic energy, (ii) electron-nucleus interaction potential, (iii) electron-electron direct interaction potential and (iv) electronelectron exchange interaction within the WS cells. The kinetic energy part for electrons at a particular point $(r)$ within the WS cell is given by

$$
E_{K E}(x)=\int_{r_{n}}^{r} d^{3} r \frac{e B}{2 \pi^{2}} \sum_{\nu=0}^{\nu_{\max }(r)}\left(2-\delta_{\nu 0}\right) \int_{0}^{p_{F}(r)} d p_{z}\left[\left(p_{z}^{2}+m_{\nu}^{2}\right)^{1 / 2}-m_{e}\right]
$$

Evaluating the integral over $p_{z}$ analytically and then substituting for $p_{F}(x)$ from eqn.(11), with the fitted form of $\phi(x)$, one can obtain the electron kinetic energy $E_{K E}$, as a function of $r$ or $x$ by the numerical evaluation of above integral over $r$ or $x$ respectively. We have seen that within the WS cells, for a particular value of $B$, the kinetic energy part satisfies a power law of the form $E_{K E}=\alpha x^{\beta}$, where the parameters $\alpha$ and $\beta$ are functions of magnetic field strength. In TABLE-I we have shown these variations. The change is more significant for $\alpha$ than $\beta$. Therefore, just like the electron density, the electron kinetic energy is also an increasing function of radial coordinate $x$.

Next we consider the three possible types of interaction potential. Let us first consider the electron-nucleus interaction part, given by [13]

$$
\begin{gathered}
E_{e n}(r)=-Z e^{2} \int_{r_{n}}^{r} d^{3} r \frac{n_{e}}{r} \\
=-4 \pi Z e^{2} \mu^{2} \int_{x_{n}}^{x} x d x n_{e}(x)
\end{gathered}
$$

To obtain the electron-nucleus interaction energy within the WS cell for a constant $B$, we substitute the expression for electron number density $n_{e}(x)$ from eqn.(10), and then 
numerically integrate over $x$ In fig.(4) we have shown the variation of $E_{\text {en }}(x)=\left|E_{\text {en }}(x)\right|$ as a function of $x$ within the WS cell for three different magnetic field strengths: $B=$ $B_{c}^{(e)}, 10^{2} \times B_{c}^{(e)}$ and $10^{3} \times B_{c}^{(e)}$. For very low $x$ values, i.e., almost on the surface of the nucleus at the centre, the magnitude of the potential energy is an increasing function of $x$, which actually means that the region is strongly attractive in nature. Again at the surface region, just at the skin of the cell, it is again an increasing function of $x$ (with larger gradient), so that the attractive field again becomes extremely strong at the surface region to keep the outer most electrons confined within the cell. At the middle region, there is a kind of saturation and force due to electron-nucleus interaction almost vanishes. This is quite analogous to the normal metallic scenario, in which electric field can not exist.

Next we consider the electron-electron interaction. Let us first evaluate the direct term. It is given by [13]

$$
E_{e e}^{(d)}=\frac{1}{2} e^{2} \int d^{3} r n_{e}(r) \int d^{3} r^{\prime} n_{e}\left(r^{\prime}\right) \frac{1}{\left|\vec{r}-\overrightarrow{r^{\prime}}\right|}
$$

Assuming $\vec{r}$ as the principal axis and $\theta$ is the angle between $\vec{r}$ and $\overrightarrow{r^{\prime}}$, we have $d^{3} r=4 \pi r^{2} d r$, $d^{3} r^{\prime}=2 \pi r^{\prime 2} d r^{\prime} \sin \theta d \theta$ (we have assumed that the vectors $\vec{r}$ and $\overrightarrow{r^{\prime}}$ are on the same plane) and $\left|\vec{r}-\overrightarrow{r^{\prime}}\right|=\left(r^{2}+r^{\prime 2}-2 r r^{\prime} \cos \theta\right)^{1 / 2}$. The limits for both $r$ and $r^{\prime}$ are from $r_{n}$ to $r_{s}$ and the range of $\theta$ is from 0 to $\pi$. Let us first evaluate the angular integral, given by

$$
I\left(r, r^{\prime}\right)=\int_{0}^{\pi} \frac{\sin \theta}{\left(r^{2}+r^{\prime 2}-2 r r^{\prime} \cos \theta\right)^{1 / 2}}
$$

It is straight forward to show that $I\left(r, r^{\prime}\right)=\left(r+r^{\prime}\right)-\left|r-r^{\prime}\right|$. Then we can express the electron-electron direct part in the form

$$
E_{e e}^{(d)}=4 \pi^{2} e^{2} \int_{r_{n}}^{r} r d r n_{e}(r) \int_{r_{n}}^{r} r^{\prime} d r^{\prime} n_{e}\left(r^{\prime}\right)\left[\left(r+r^{\prime}\right)-\left|r-r^{\prime}\right|\right]
$$

Now from this equation it is trivial to show that the quantity within the third bracket will be $2 r^{\prime}$ for $r^{\prime}<r$ and for the opposite case it will be $2 r$. Then the above expression for direct interaction, reduces to the following simple form:

$$
\begin{aligned}
E_{e e}^{(d)} & =8 e^{2} \pi^{2}\left\{\int_{r_{n}}^{r} r d r n_{e}(r) \int_{r_{n}}^{r} r^{\prime 2} d r^{\prime} n_{e}\left(r^{\prime}\right)\right. \\
& \left.+\int_{r_{n}}^{r} r^{2} d r n_{e}(r) \int_{r}^{r_{s}} r^{\prime} d r^{\prime} n_{e}\left(r^{\prime}\right)\right\}
\end{aligned}
$$

Following the same procedure adopted for $e-n$ case, we have evaluated numerically the above coupled integrals. In fig.(5) we have shown the variation of $E_{e e}^{d}(x)$ within the WS cell 
for three different magnetic field strengths: $B=B_{c}^{(e)}, 10^{2} \times B_{c}^{(e)}$ and $10^{3} \times B_{c}^{(e)}$. Surprisingly, the variations are almost identical with $\left|E_{e n}\right|$. However, the magnitudes are several orders less than the corresponding electron-nucleus part. Of course, unlike the $E_{e n}$, the direct term is positive throughout and for all the values of magnetic field strength.

Next we shall consider the exchange term, which is negative in nature. The magnitude of exchange energy integral corresponding to the $i$ th. electron in the cell is given by [13]

$$
E_{e e}^{(e x)}=\frac{e^{2}}{2} \sum_{j} \int d^{3} r d^{3} r^{\prime} \frac{1}{\left|\vec{r}-\overrightarrow{r^{\prime}}\right|} \bar{\psi}_{i}(\vec{r}) \bar{\psi}_{j}\left(\overrightarrow{r^{\prime}}\right) \psi_{j}(\vec{r}) \psi_{i}\left(\overrightarrow{r^{\prime}}\right)
$$

where the spinor wave function $\psi(\vec{r})$ is given by eqns.(2)-(5) in [13] and $\bar{\psi}(\vec{r})=\psi^{\dagger}(\vec{r}) \gamma_{0}$, the adjoint of the spinor and $\gamma_{0}$ is the zeroth part of the Dirac gamma matrices $\gamma_{\mu}$. Now it is very easy to show that for $t=t^{\prime}$

$$
\begin{aligned}
\bar{\psi}_{i}(\vec{r}) \psi_{i}\left(\overrightarrow{r^{\prime}}\right)= & \frac{2 m}{L_{y} L_{z} E_{\nu}} \exp \left[-i\left\{p_{y}\left(y-y^{\prime}\right)+p_{z}\left(z-z^{\prime}\right)\right\}\right] \\
& \left\{I_{\nu ; p_{y}}(x) I_{\nu ; p_{y}}\left(x^{\prime}\right)+I_{\nu-1 ; p_{y}}(x) I_{\nu-1 ; p_{y}}\left(x^{\prime}\right)\right\}
\end{aligned}
$$

Similarly, we have

$$
\begin{aligned}
\bar{\psi}_{j}\left(\overrightarrow{r^{\prime}}\right) \psi_{j}(\vec{r})= & \frac{2 m}{L_{y} L_{z} E_{\nu}^{\prime}} \exp \left[i\left\{p_{y}^{\prime}\left(y-y^{\prime}\right)+p_{z}^{\prime}\left(z-z^{\prime}\right)\right\}\right] \\
& \left\{I_{\nu^{\prime} ; p_{y}^{\prime}}(x) I_{\nu^{\prime} ; p_{y}^{\prime}}\left(x^{\prime}\right)+I_{\nu^{\prime}-1 ; p_{y}^{\prime}}(x) I_{\nu^{\prime}-1 ; p_{y}^{\prime}}\left(x^{\prime}\right)\right\}
\end{aligned}
$$

where $I_{\nu ; p_{y}}(x)$ is same as $I_{\nu}$, given by eqn.(5) in [13]. When these two terms are combined, we have, after replacing the sum over $j$ by the integrals

$$
\begin{aligned}
& L_{y} L_{z} \int_{-\infty}^{+\infty} d p_{y}^{\prime} \int_{-p_{F}}^{+p_{F}} d p_{z}^{\prime} \\
& E_{e e}^{(e x)}=\left(\frac{e^{2}}{2}\right)\left(\frac{4 m^{2}}{L_{y}^{2} L_{z}^{2} E_{\nu}}\right) \sum_{\nu^{\prime}=0}^{\nu_{\max }}\left(2-\delta_{\nu^{\prime} 0}\right) \int \ldots \int L_{y} d p_{y}^{\prime} L_{z} d p_{z}^{\prime} d^{3} r d^{3} r^{\prime} \frac{1}{E_{\nu^{\prime}}} \frac{1}{\left|\vec{r}-\overrightarrow{r^{\prime}}\right|} \\
& \exp \left[-i\left\{\left(p_{y}-p_{y}^{\prime}\right)\left(y-y^{\prime}\right)+\left(p_{z}-p_{z}^{\prime}\right)\left(z-z^{\prime}\right)\right\}\right] \\
& {\left[\left\{I_{\nu ; p_{y}}(x) I_{\nu ; p_{y}}\left(x^{\prime}\right)+I_{\nu-1 ; p_{y}}(x) I_{\nu-1 ; p_{y}}\left(x^{\prime}\right)\right\}\right.} \\
& \left.\left\{I_{\nu^{\prime} ; p_{y}^{\prime}}(x) I_{\nu^{\prime} ; p_{y}^{\prime}}\left(x^{\prime}\right)+I_{\nu^{\prime}-1 ; p_{y}^{\prime}}(x) I_{\nu^{\prime}-1 ; p_{y}^{\prime}}\left(x^{\prime}\right)\right\}\right]
\end{aligned}
$$

It is possible to evaluate the integrals over $y^{\prime}$ and $z^{\prime}$, given by [22]

$$
\begin{aligned}
\int_{-\infty}^{+\infty} \int_{-\infty}^{+\infty} d y^{\prime} d z^{\prime} \frac{1}{\mid \vec{r}-\overrightarrow{r^{\prime} \mid}} & \quad \exp \left[-i\left\{\left(p_{y}-p_{y^{\prime}}\right)\left(y-y^{\prime}\right)+\left(p_{z}-p_{z^{\prime}}\right)\left(z-z^{\prime}\right)\right\}\right] \\
= & \frac{4 \pi}{2 K} \exp \left(-K\left|x-x^{\prime}\right|\right)
\end{aligned}
$$


where $K=\left[\left(p_{y}-p_{y^{\prime}}\right)^{2}+\left(p_{z}-p_{z^{\prime}}\right)^{2}\right]^{1 / 2}$. Further, the integral over $y$ and $z$ is given by

$$
\int_{-\infty}^{+\infty} \int_{-\infty}^{+\infty} d y d z=L_{y} L_{z}
$$

Since the final form of exchange integral is multi-dimensional in nature with an extremely complicated structure of integrand, it is absolutely impossible to have analytical solution for $e-e$ exchange. We have therefore adopted the multi-dimensional Monte-Carlo integration code to evaluate the exchange term. It is found the variation of the magnitude of exchange energy with the scaled radius $x$ within the WS cells for a constant $B$ can be expressed by the power law formula, given by

$$
E_{e e}^{(\mathrm{ex})}=p x^{q}
$$

where the parameters $p$ and $q$ are again functions of magnetic field strength $B$. The variation of the parameters $p$ and $q$ with magnetic field are shown in TABLE-I.

From the variation of the parameters $p$ and $q$ with $B$, it is quite clear that the magnitude of exchange energy also increases with $x$ and also with the magnetic field strength $B$, i.e., minimum at the central nuclear surface and maximum at the WS cell boundary.

The total energy of an electron at any point $x$ within the cell is then given by (with negative values for exchange energy)

$$
E_{t o t}=E_{K E}+E_{e n}+E_{e e}^{(\mathrm{d})}+E_{e e}^{(\mathrm{ex})}
$$

We have seen that at the central part up to certain radial distance $r_{c}$ from the centre of the WS cells, the total energy is negative, whereas beyond this radial distance and up to the WS cell surface, it is positive. We have evaluated numerically the $x\left(=x_{c}\right.$ say), the value of the point within the cell at which the total energy of the electrons just become zero for a given magnetic field. The position $x_{c}$ of this zero energy point changes with the strength of magnetic field and is shown in fig.(6). Further, this variation can also be expressed by the functional form, given by

$$
x_{c}=\alpha+\beta \exp \left(\gamma \frac{B}{B_{c}^{(e)}}\right) \mathrm{MeV}^{-1}
$$

with $\alpha=0.5, \beta=1.34$ and $\gamma=0.205$. If the total energy for the electrons at the central part is found to be negative, then obviously, we can not expect that all the $Z$ electrons in the cell particularly near the central region, are participating in statistical process. Some of 
them remain bound in negative energy states near the nuclear surface at the centre of the WS cell.

The overall pressure term can also be obtained for the electron gas from the total energy $E_{\text {tot }}$, given by

$$
P(x)=n_{e}^{2}(x) \frac{\partial E_{t o t}}{\partial n_{e}}=P_{k}(x)+P_{c}(x)
$$

where

$$
P_{k}=\frac{e B}{2 \pi^{2}} \sum_{\nu=0}^{\nu_{\max }}\left(2-\delta_{\nu 0}\right) \int_{0}^{p_{F}} \frac{p_{z}^{2}}{\left(p_{z}^{2}+m_{\nu}^{2}\right)^{1 / 2}} d p_{z}
$$

is the electron kinetic pressure part. The momentum integral for the kinetic pressure can very easily be obtained, and is given by

$$
P_{k}=\frac{e B}{2 \pi^{2}} \sum_{\nu=0}^{\nu_{\max }}\left(2-\delta_{\nu 0}\right)\left[p_{F}\left(p_{F}^{2}+m_{\nu}^{2}\right)^{1 / 2}-m_{\nu}^{2} \ln \left(\frac{p_{F}+\left(p_{F}^{2}+m_{\nu}^{2}\right)^{1 / 2}}{m_{\nu}}\right)\right]
$$

with $p_{F}=p_{F}(x)$. This expression gives the electron kinetic pressure at various points within the WS cell. The other term, $P_{c}$, coming from the interaction part, $E_{c}=E_{e n}+E_{e e}^{(d)}+E_{e e}^{(e x)}$, and is given by

$$
P_{c}(x)=n_{e}^{2}(x) \frac{\partial E_{c}}{\partial n_{e}}
$$

The interaction part of electron pressure is obtained numerically by substituting the expression for $p_{F}(x)$ and evaluating the derivatives over $n_{e}$ numerically for a given $B$, at a particular point $x$ within the cell and also for a given set of $A$ and $Z$. We have noticed that at the central region of WS cells, since the density dominates over the magnetic field part, the total pressure is positive, while at the outer end, the surface region, since magnetic field dominates over the density contribution, it becomes negative. It is found numerically that at some point within the WS cell for a given $B$, the total pressure becomes exactly zero. It is found that this critical position is a function of magnetic field strength and decreases with the increase in magnetic field strength. In fig.(7) we have plotted this variation. The functional dependence can also be expressed as

$$
x_{c}=\alpha+\beta \exp \left[-\gamma \frac{B}{B_{c}^{(e)}}\right] \quad \mathrm{MeV}^{-1}
$$

with $\alpha=0.38, \beta=1.28$ and $\gamma=0.171$. The qualitative form of this variation is exactly identical with that of $x_{c}$ for total energy. 


\section{INNER CRUST MATTER}

In this section we shall study the effect of strong quantizing magnetic field on dense nuclear matter composed of nuclei, free neutrons and electrons at sub-nuclear density. In the conventional neutron star model, it is generally believed that for a typical neutron star of radius $\approx 10 \mathrm{~km}$, the width of inner crust is about $0.5-0.6 \mathrm{~km}$. The constituents are a mixture of heavy nuclei (of which some of them are highly neutron rich), electron gas and there are also free neutrons (if the matter density is above the neutron drip point). It is also believed that the free neutrons in the inner crust region may be in super-fluid state. The density range of this zone depends on the type of equation of state or the mass formula for the heavy nuclei, including the highly neutron rich nuclei. The density range in the conventional picture is $\sim 10^{9}-10^{12} \mathrm{gm} / \mathrm{cm}^{3}$. The inner crust is covered by the outer crust and is mainly the crystalline structure of compressed solid crystal of fully ionized metallic iron immersed in a dense electron gas. In the previous section we have made a detailed study of various aspects of this sub-nuclear matter in the outer crust region. For a typical neutron star, the width of the outer crust region is $\sim 0.2-0.4 \mathrm{Km}$. Analogous to the model calculation of the outer crust matter, which is assumed to be a regularly arranged structure of WS cells, for the study of inner crust region, we have assumed that the matter is a mixture of WS cells of not only metallic iron, but a large number of neutron rich nuclei and a gas of free neutrons. We further assume that the free neutrons in this region, above the neutron drip point are in normal fluid state. The effect of strong quantizing magnetic field on the super-fluidity of free neutron matter, in which magnetic field interacts with the anomalous magnetic moment of neutrons, will be studied in future.

In this section, to study the effect of strong quantizing magnetic field on inner crust matter, we consider two types of conventional nuclear mass formulae, which are generally used below the nuclear saturation density, but applicable near neutron drip region. These two mass formulae are (i) Harrison-Wheeler (HW) equation of state, and (ii) Bethe-BaymPethick (BBP) equation of state [14, 15, 16].

In HW equation of state, the relativistic electrons are assumed to be in $\beta$-equilibrium with the nuclei (which also includes the neutron rich nuclei). Whereas, above the neutron drip density, the $\beta$-equilibrium is among the nuclei, free neutrons and the electrons. In this article, we assume $\beta$-equilibrium configuration in presence of a strong quantizing back ground 
magnetic field. In this section, the properties of inner crust matter have been investigated in presence of such a strong magnetic field. In the conventional physical picture, in presence of free electron gas in the medium, the balance between the Coulomb force and the nuclear force, which gives $F e^{56}$ as the most stable nucleus will get shifted towards the heavier nuclei. The nuclei, formed in such an environment will contain more neutrons (by inverse $\beta$-decay) compared to the usual picture. The Coulomb force plays a very little role. The nuclei becomes more and more neutron rich as the electron density goes up and when the matter density increases to $\sim 4 \times 10^{11} \mathrm{gm} / \mathrm{cm}^{3}$ in the inner crust region, the ratio $n / p$ reaches a critical level. Any further increase in density will lead to neutron drip in the medium. In such a scenario, highly neutron rich nuclei, electrons and free neutrons co-exist in chemical equilibrium. Again, with the increase in matter density, beyond neutron drip density, free neutrons appear in the medium and at some stage the kinetic pressure of the system will be dominated by the free neutron pressure. As we shall see that this positive pressure contribution of free neutron gas will make the total pressure within the inner crust region a positive definite, even in presence of strong magnetic field. Whereas in the outer crust, the total pressure, coming from the electron gas only and is negative at the surface region of WS cells in presence of strong magnetic field. Just like the outer crust region, we have assumed that only electron part of inner crust gets affected by the strong magnetic field. We have not considered the effect of quantizing magnetic field on bound protons and neutrons and above neutron drip density, on free neutrons. We have noticed that the presence of strong magnetic field makes a lot of qualitative and quantitative changes in the physical and chemical properties of inner crust matter.

The BBP equation of state is applicable for nuclear matter in the density range from neutron drip density $\rho_{\text {drip }}$ to normal nuclear density $\rho_{\text {nuc. }}$. Here also the matter is assumed to be composed of nuclei $\left(F e^{56}\right.$ and other neutron rich nuclei beyond $F e^{56}$ ), electrons and free neutrons. It is well known that BBP equation of state is a considerable improvement over the HW equation of state. In BBP equation of state, a mass formula, more or less like the HW equation of state is used but incorporated a lot of improvements from detailed many body calculation. The nuclear surface energy, for example, assumed in the previous treatment to be that of a nucleus in vacuum. An introduction of free neutron gas out side the nuclei reduces the nuclear surface energy. This is quite correct, because when inside and out side of a nuclei become identical, the surface energy must vanish. In BBP equation of state, 
the nuclear Coulomb energy is included in a more accurate manner and is called nuclear lattice Coulomb energy. It is well known that the BBP equation of state is applicable up to nuclear density, therefore, when all the bound nuclei dissolves into a continuous matter, mainly composed of free neutrons and a tiny fraction of protons and electrons, to incorporate this type of melting process of nuclei, in the BBP equation of state the factors giving the fractional volume occupied by the nuclei and the fractional volume occupied by the free neutron gas have been taken into account. It is also assumed that at this density $\left(<\rho_{\text {nuc. }}\right)$ the nuclei are stable to $\beta$-decay. Further, the neutrons in the free neutron gas are in chemical equilibrium with the electrons and the nucleons within the nuclei and in addition to the $\beta$ stability of the nuclei, the whole system must be in chemical equilibrium. In this model the kinetic pressure of free neutrons must necessarily be equal with the pressure of the nucleons bound within the nuclei. This is the condition for mechanical equilibrium. In our present calculation, with this BBP equation of state, we have investigated some of the interesting properties of neutron star inner crust matter in presence of strong quantizing magnetic field, assuming that only the electron part gets affected by the presence of strong magnetic field. Since detailed mathematical formalism for both HW and BBP equation of states, which are not affected by strong magnetic fields, are available in a large number of classic papers, including the original ones, and also included in many standard text books [14, 15, 16], we are therefore do not feel the need to include those mathematical steps in this article. Further, the effect of strong quantizing magnetic field on the electron part has already been discussed in the previous section. In this section, we therefore present only the numerical results based on these two equation of states and the results related to electron gas as given in the previous section.

In the numerical computation, we have found that for very low mass number, since there is no free neutrons available in the system, even much above the neutron drip density, only electrons contribute in total kinetic pressure and is negative. This result is almost identical with the outer crust scenario. In fig.(8) we have plotted the variation of the critical value of mass number $A$ and the corresponding atomic number $Z$ with the strength of magnetic field, using HW equation of state for the nuclei and for the electrons, for which the resukts are given in the previous section. These are the critical values at which the kinetic pressure of the inner crust region just becomes zero, i.e., the system just becomes mechanically stable. It is obvious from this figure that the minimum of mass number of the nuclei for which the inner 
crust matter just becomes mechanically stable, increases with the strength of magnetic field. In other words, this is equivalent to say that the presence of strong magnetic field makes the nuclei more and more massive or increases the minimum mass of the nuclei in the inner crust region. In some of our previous work, done long ago [12], we have seen that the same conclusion is applicable for quark matter. The presence of strong quantizing magnetic field generates quark mass dynamically in dense quark matter composed of massless quarks. This is the well known magnetic field induced chiral symmetry violation. The strong quantizing magnetic field acts like a catalyst to generate / increase mass. In fig.(9) we have plotted the same kind of variation using the BBP equation of state. In this case the minimum mass of the nuclei are more than that obtained from HW equation of state. Further, one should notice from these two figures (figs.(8) and (9)) that for low and moderate strength of magnetic field, the effect is not so significant. But beyond $10^{15} \mathrm{G}$, when all the electrons occupy the zeroth Landau level, or in other words, when the quantum mechanical effect of strong magnetic field is most important, the minimum mass rises sharply to very large values. One can see from [12] that the qualitative nature of the curve showing the dependence of dynamical quark mass on the magnetic field strength is exactly identical, although the physical scenarios are completely different.

In fig.(10), we have plotted the variation of the ratio $n_{e} / n$ and $n_{n} / n$ with the mass number using HW equation of state. Here $n_{e}$ is the electron density, $n_{n}$ is the free neutron density and $n=n_{n}+n_{e} A / Z$ is the total baryon density of inner crust matter. Dashed curve is for $B=0$, middle and the lower curves, indicated by $e 1$ and $e 2$ are for $B=10^{2} B_{c}^{(e)}$ and $10^{3} B_{c}^{(e)}$ respectively. The curve indicated by $n$ is for free neutron gas. In the HW equation of state, the neutron number density above the neutron drip point is independent of magnetic field strength. Further, the mass number around which neutrons are liberated from neutron rich nuclei is about 95, which is again independent of magnetic field strength. However, immediately after the emission of neutrons from heavy neutron rich nuclei the overall kinetic pressure can not become non-zero. This is also quite clear from the figs.(8) and (9). In fig.(11), we have plotted the same kind of variations as shown in fig.(10). Here we have used the BBP equation of state. Solid curves are for $B=0$, while the dashed curves are for $10^{3} B_{c}^{(e)}$. In this case the neutron drip out from heavy neutron rich nuclei around $A=100$, which is little bit heavier than the HW case. Further, the qualitative nature of $x_{n}=n_{n} / n$ is totally different from HW case. Instead of increase initially and then saturates, 
as we observe in HW case, it decreases monotonically with A. Also, the free neutron density very weakly depends on the strength of magnetic field. These qualitative differences are found to be the consequence of chemical equilibrium among the free neutrons, electrons and nucleons within the heavy neutron rich nuclei and the overall $\beta$-equilibrium condition.

In fig.(12). the variation of total nuclear density is plotted against the mass number from HW equation of state for four different magnetic field strengths: $B=0, B=10 B_{c}^{(e)}$, $B=10^{2} B_{c}^{(e)}$ and $B=10^{3} B_{c}^{(e)}$, indicated in the diagram by $0,1,10^{2}$ and $10^{3}$ respectively. Initially, for low $A$ values, only the bound neuclons within the nuclei $\left(=A n_{e} / Z\right)$ contributes. Just beyond the critical value, $A=95$, since neutrons drip out from the heavy neutron rich nuclei, the number density jumps suddenly. Of course, below $A=60$, we can not have stable inner crust matter. In fig.(13), we have plotted the same kind of variation for BBP equation of state. In this case the qualitative and the quantitative changes are again because of the chemical equilibrium conditions

The equation of state from HW mass formula is plotted in fig.(14). Here the lower curve is for $B=0$, middle and upper curves are for $B=B_{c}^{(e)}$ and $B=10^{3} B_{c}^{e)}$ respectively. In fig.(15), we have plotted the same kind variation from BBP equation of state.

From these two figures it is quite obvious that in presence of strong quantizing magnetic field, the inner crust matter becomes mechanically stable only at very high density, when enough number of free neutrons are available in the system. In this high density situation, positive neutron kinetic pressure, dominates over negative electron pressure, in presence of strong quantizing magnetic field. This is true for both HW and BBP equation of states. Further, it is also obvious from the figures, that the matter becomes softer with the increase in magnetic field strength. The qualitative nature of both the equation of states are almost identical in presence of strong magnetic field. However, the HW equation of state is a bit softer than the BBP case.

\section{CONCLUSIONS}

In the first part of this article we have presented our investigation on the properties of dense outer crust matter of the magnetars. We have replaced the dense metallic iron crystal by a regular array of spherically symmetric WS cells. It has been observed that the radius of each cell decreases with the magnetic field strength. In this article, however, we have 
not considered the magnetic field induced deformation of WS cells, which is quite likely if the magnetic field is strong enough. In future we shall present this important issue with a completely different approach.

It has also been noticed that the upper limit of Landau quantum number is a function of positional coordinate of the electron with which it is associated within the WS cells. We have observed that at the surface region, for all the values of magnetic field strength, this upper limit becomes identically zero. Which actually means that the electrons near the WS cell surface are strongly polarized in the opposite direction of external magnetic field. Whereas, for $B>10^{15} \mathrm{G}$, they are polarized at every points within the cells.

It has been observed that the electron density within the cells increases with the increase in magnetic field strength. Further, for all the values of magnetic field strength, the electron density is maximum near the nuclear surface $\left(r=r_{n}\right)$ and minimum at the WS cell boundary $\left(r=r_{s}\right)$.

We have also studied the variations of kinetic energy, electron-nucleus interaction energy, electron-electron direct potential energy and electron-electron exchange interaction part. We have shown the variation of these quantities within the WS cells for a number of magnetic field strengths. Interestingly, we have noticed that the total energy of the electrons is negative near the nuclear surface within the cells and becomes positive beyond some radial distance $\left(x_{c}\right)$ which increases with the strength of magnetic field at low and moderate region but saturates to some constant value for $B \geq 10^{15} \mathrm{G}$, when all the electrons occupy their zeroth Landau level. Hence we can not conclude that all the $Z$ electrons in the cell are participating in statistical process. Some of them remain bound in negative energy orbitals near the nuclear surface at the centre of the WS cell.

In the second part of this work, we have investigated some of the properties of inner crust matter of magnetars. We have used HW and BBP equation of states for the nuclear mass formula. It has been observed from both the models, that for a stable inner crust matter, the nuclei present must be heavier than iron and much more neutron rich. The heaviness is more in the case of BBP equation of state. We have also noticed that for low and moderate values of magnetic field strength, the variation of mass number and the corresponding atomic number with magnetic field is not so significant. Whereas, for $B \geq 10^{15} \mathrm{G}$, when electrons occupy only the zeroth Landau level, then much more heavier neutron rich nuclei are formed in the inner crust region. It is found that high magnetic field behaves like a catalyst to 
generates heavy neutron rich nuclei.

We have observed that initially the electron density increases with the increase in mass number, but as soon as free neutrons appear in the system, the electron density decreases and saturates to some constant value which depends on the magnetic field strength. In the case of HW equation of state, free neutron density does not depend on the strength of magnetic field, whereas, for BBP case, because of chemical equilibrium condition, the free neutron density depends very weakly on the magnetic field strength. We have noticed that in the case of BBP equation of state the overall qualitative difference is because of chemical equilibrium among the constituents.

The total baryon density rises sharply like an avalanche for the value of $A$ at which free neutrons appear in the system. However, for BBP equation of state, because of chemical equilibrium condition, the rise is not so sharply visible for a given magnetic field $B$.

The qualitative nature of equation of states are almost identical. It is found that in presence of strong magnetic field, the inner crust matter becomes mechanically stable (with the positive value of kinetic pressure) only at very high density.

We therefore believe that to study various properties of dense matter associated with magnetars, one must consider all these significant changes from the conventional scenario. Further, we expect that the strong magnetic field, if present well within the inner crust of magnetars, must affect the super-fluidity of cold neutron matter.

[1] R.C. Duncan and C. Thompson, Astrophys. J. Lett. 392, L9 (1992); C. Thompson and R.C. Duncan, Astrophys. J. 408, 194 (1993); C. Thompson and R.C. Duncan, MNRAS 275, 255 (1995); C. Thompson and R.C. Duncan, Astrophys. J. 473, 322 (1996).

[2] P.M. Woods et. al., Astrophys. J. Lett. 519, L139 (1999); C. Kouveliotou, et. al., Nature 391, 235 (1999).

[3] K. Hurley, et. al., Astrophys. Jour. 442, L111 (1999).

[4] S. Mereghetti and L. Stella, Astrophys. Jour. 442, L17 (1999); J. van Paradihs, R.E. Taam and E.P.J. van den Heuvel, Astron. Astrophys. 299, L41 (1995); S. Mereghetti, astro-ph/99111252; see also A. Reisenegger, astro-ph/0100301]; see also S. Mereghetti, arXiv:0904.4880v1, for current status on the observational aspects of magnetars. 
[5] D. Bandopadhyaya, S. Chakrabarty, P. Dey and S. Pal, Phys. Rev. D58, (1998), 121301.

[6] S. Chakrabarty, D. Bandopadhyay and S. Pal, Phys. Rev. Lett. 78, (1997), 2898; D. Bandopadhyay, S. Chakrabarty and S. Pal, Phys. Rev. Lett. 79, (1997), 2176.

[7] C.Y. Cardall, M. Prakash and J.M. Lattimer, astro-ph/0011148 and references therein; L.B. Leinson and A. Pérez, astro-ph/9711216; D.G. Yakovlev and A.D. Kaminkar, The Equation of States in Astrophysics, eds. G. Chabrier and E. Schatzman P.214, Cambridge Univ; S. Chakrabarty and P.K. Sahu, Phys. Rev. D53, (1996), 4687; S. Ghosh, S. Mandal and S. Chakrabarty, Ann. Phys. 312 (2004) 398; S. Mandal, R. Saha, S. Ghosh and S. Chakrabarty, Phys. Rev. C74, (2006), 015801.

[8] S. Chakrabarty, Phys. Rev. D51 (1995) 4591; T. Ghosh and S. Chakrabarty, Phys. Rev. D63 (2001) 043006; T. Ghosh and S. Chakrabarty, Int. Jour. Mod. Phys. D10 (2001) 89;

[9] Sutapa Ghosh, Sanchayita Ghosh, Kanupriya Goswami, Somenath Chakrabarty, Ashok Goyal, Int. Jour. Mod. Phys. D11 (2002) 843; Sutapa Ghosh and Somenath Chakrabarty, Mod. Phys. Lett. A17, (2002) 2147.

[10] V.P. Gusynin, V.A. Miransky and I.A. Shovkovy, Phys. Rev. D52, 4747 (1995); D.M. Gitman, S.D. Odintsov and Yu.I. Shil'nov, Phys. Rev. D54, 2968 (1996); D.S. Lee, C.N. Leung and Y.J. Ng, Phys. Rev. D55, 6504 (1997); V.P. Gusynin and I.A. Shovkovy, Phys. Rev. D56, 5251 (1995).

[11] D.S. Lee, C.N. Leung and Y.J. Ng, Phys. Rev. D57, 5224 (1998); V.P. Gusynin, V.A. Miransky and I.A. Shovkovy, Phys. Rev. Lett. 83, 1291 (1999); E.V. Gorbar, Phys. Lett. B491, 305 (2000); T. Inagaki, T. Muta and S.D. Odintsov, Prog. Theo. Phys. 127, 93 (1997), hep-th/9711084 T. Inagaki, S.D. Odintsov and Yu.I. Shil'nov, Int. J. Mod. Phys. A14, 481 (1999), hep-th/9709077; T. Inagaki, D. Kimura and T. Murata, Prog. Theo. Phys. 111, 371 (2004), hep-ph/0312005; V.P. Gusynin, V.A. Miransky and I.A. Shovkovy, Phys. Lett. B349, 477 (1995); S.P. Klevansky, Rev. Mod. Phys. 64, (1992) 649; C.N. Leung and S.-Y. Wang, hep-ph/0503298, hep-ph/0510066.

[12] Sutapa Ghosh, Soma Mandal and Somenath Chakrabarty, Phys. Rev. C75, (2007) 015805.

[13] Nandini Nag, Sutapa Ghosh and Somenath Chakrabarty, Ann. of Phys., 324, (2009) 499.

[14] B.K. Harrison, K.S. Yhorne, K.S. Wakano and J.A. Wheeler, Gravitation theory and gravitational collapse, University of Chicago press, Chicago, 1965.

[15] G. Baym, H.A. Bethe and C.J. Pethick, Nucl. Phys. A175, 225, (1971). 
[16] see also G. Baym, C.J. Pethick and P. Sutherland, Astrophys. Jour. 170, 299, (1971); F. Ferrini, Astr. Sp. Sci. 32, (1975), 231; A.W. Sreiner, arXiv:0711.1812v1 [nucl-th]; J. Margueron, N. Van Giai and N. Sandulescu, arXiv:0711.0106v1 [nucl-th]; S.L. Shapiro and S.A. Teukolsky, Black Holes, White Dwarfs and Neutron Stars, John Wiley and Sons, New York, (1983).

[17] E.H. Lieb and B. Simon, Phys. Rev. Lett. 31, (1973), 681; E.H. Lieb, J.P. Solovej and J. Yngvason, Phys. Rev. Lett. 69, (1992), 749; E.H. Lieb, Bull. Amer. Math. Soc., 22, (1990), 1 ;

[18] R.O. Mueller, A.R.P. Rau and L. Spruch, Phys. Rev. Lett. 26, (1971), 1136; A.R.P. Rau, R.O. Mueller and L. Spruch, Phys. Rev. A11, (1975), 1865; S.H. Hill, P.J. Grout and N.H. March, Jour. Phys. B18, (1985), 4665.

[19] R. Ruffini, "Exploring the Universe", a Festschrift in honour of Riccardo Giacconi, Advance Series in Astrophysics and Cosmology, World Scientific, Eds. H. Gursky, R. Rufini and L. Stella, Vol. 13, (2000), pp. 383; Int.Jour. of Mod. Phys. 5, (1996) 507.

[20] R.P. Feynman, N. Metropolis and E.Teller, Phy. Rev. 75, (1949), 1561.

[21] S. Chakrabarty, Phys. Rev. D54 (1996) 1306.

[22] T.D. Lee, Elementary particles and the universe: Eassays in honous of M. Gellmann, ed. John. H. Schwarz, Cambridge University Press, (1991), pp 135. 


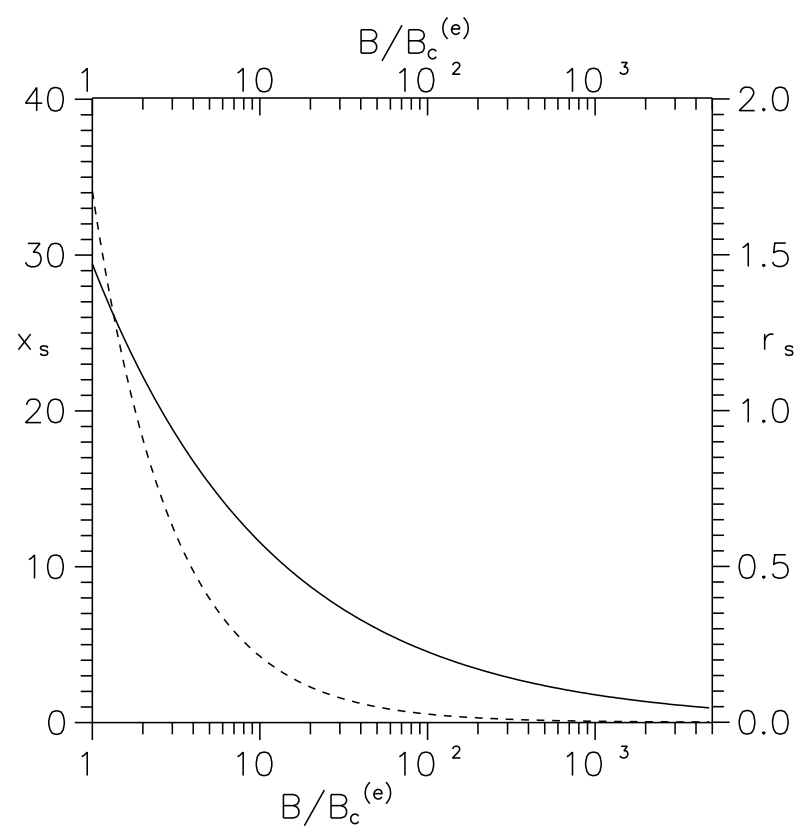

FIG. 1: The variation of scaled surface radius $x_{s}$ in $\mathrm{MeV}^{-1}$ (solid curve) and the actual radius $r_{s}$ in $\AA$ (dashed curve) with the strength of magnetic field strength (expressed in terms of $B_{c}^{(e)}$. 


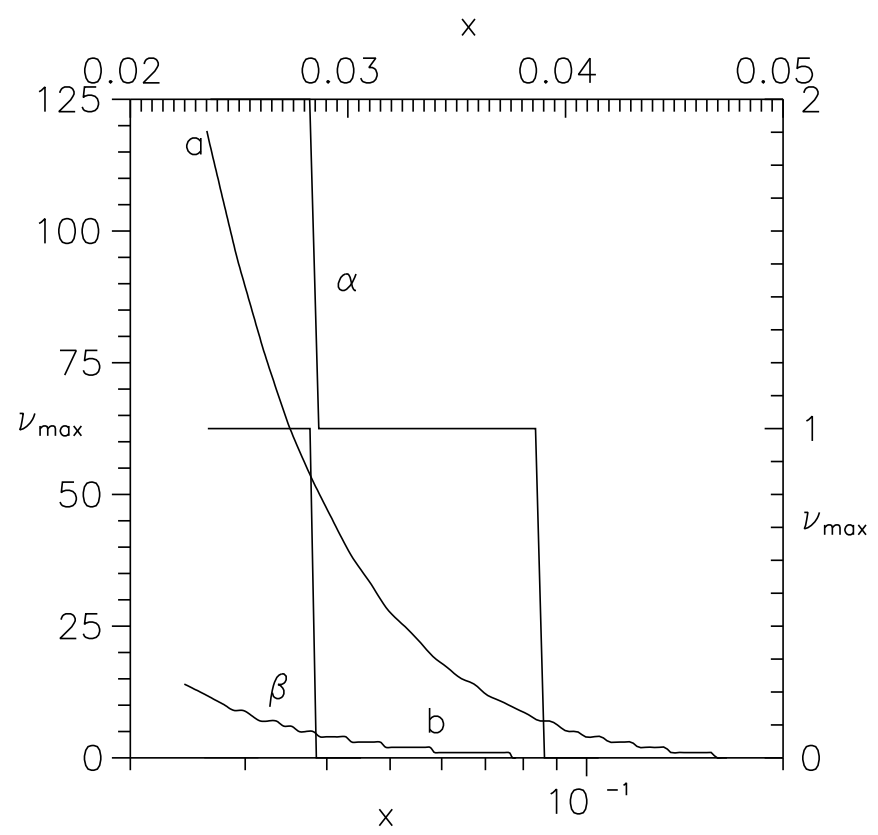

FIG. 2: The variation of $\nu_{\max }$ with $x$. For the curves $a$ and $b$, the relevant axes are at the bottom $(x)$ and at the left side $\left(\nu_{\max }\right)$, whereas for the curves $\alpha$ and $\beta$, these axes are at the top and at the right side respectively.

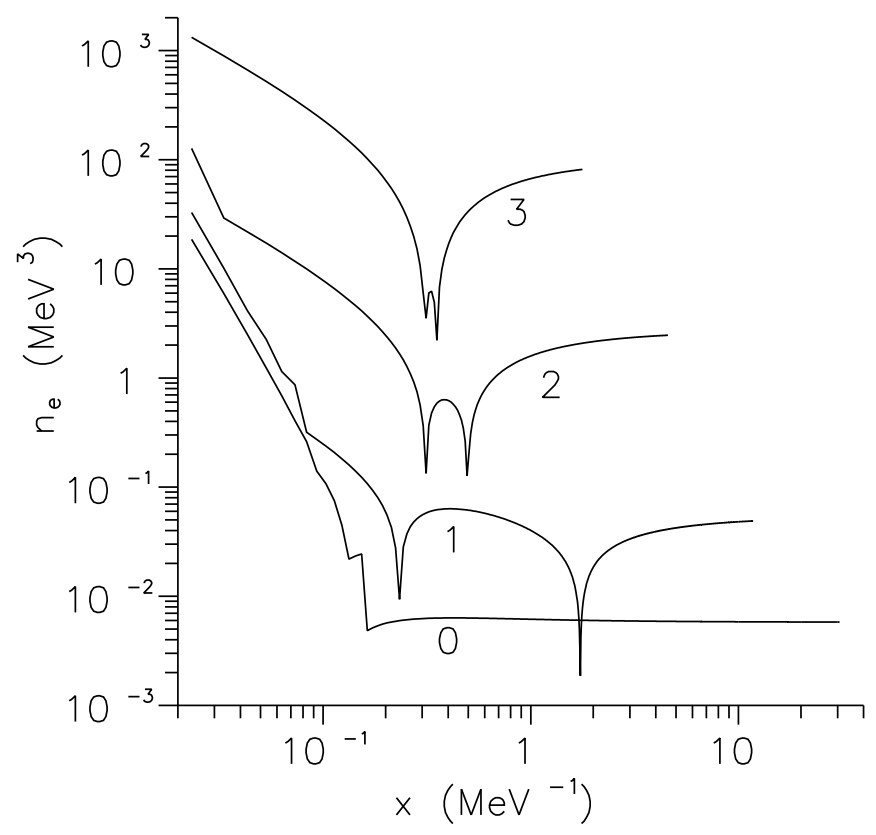

FIG. 3: The variation of electron density in $\mathrm{MeV}^{3}$ within the cells for $B=h \times B_{c}^{(e)}$ with $h=$ 1, 10,100 and 1000 indicated by the numbers $0,1,2$, and 3 . 


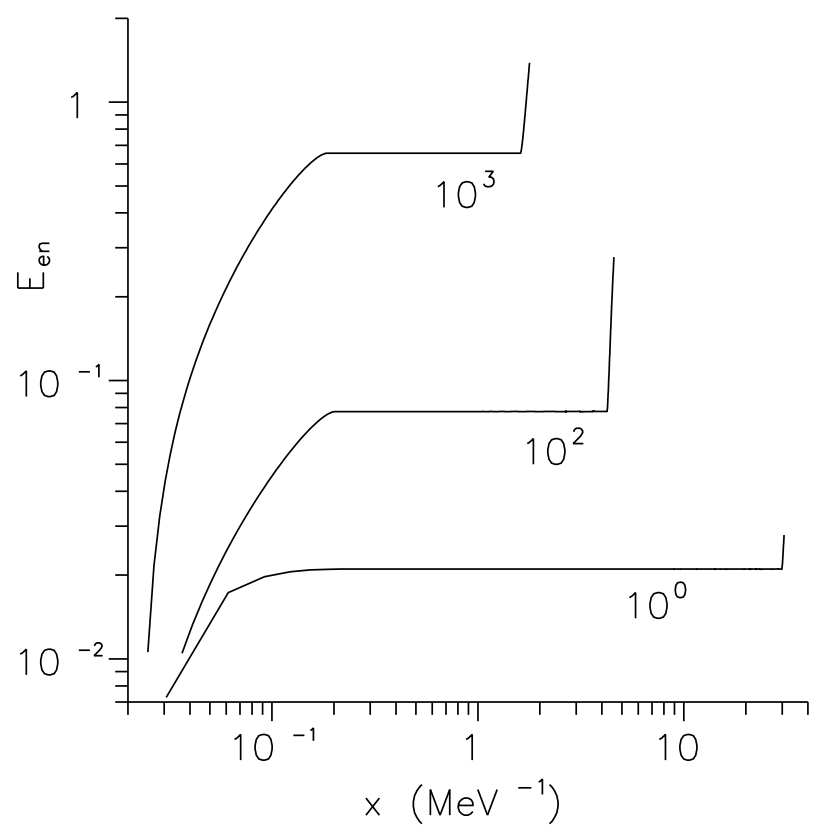

FIG. 4: The variation of the magnitude of electron-nucleus interaction energy within the WS cells for $B=h \times B_{c}^{(e)}$ with $h=1,100$ and 1000

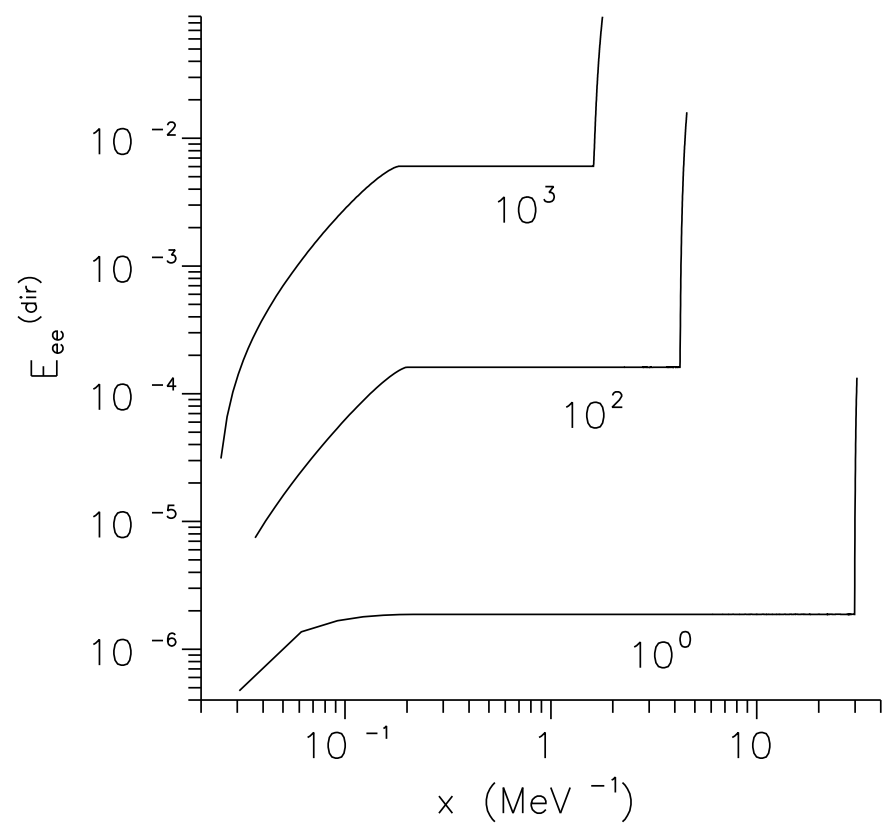

FIG. 5: The variation of electron-electron direct interaction energy within the WS cells for $B=$ $h \times B_{c}^{(e)}$ with $h=1,100$ and 1000 


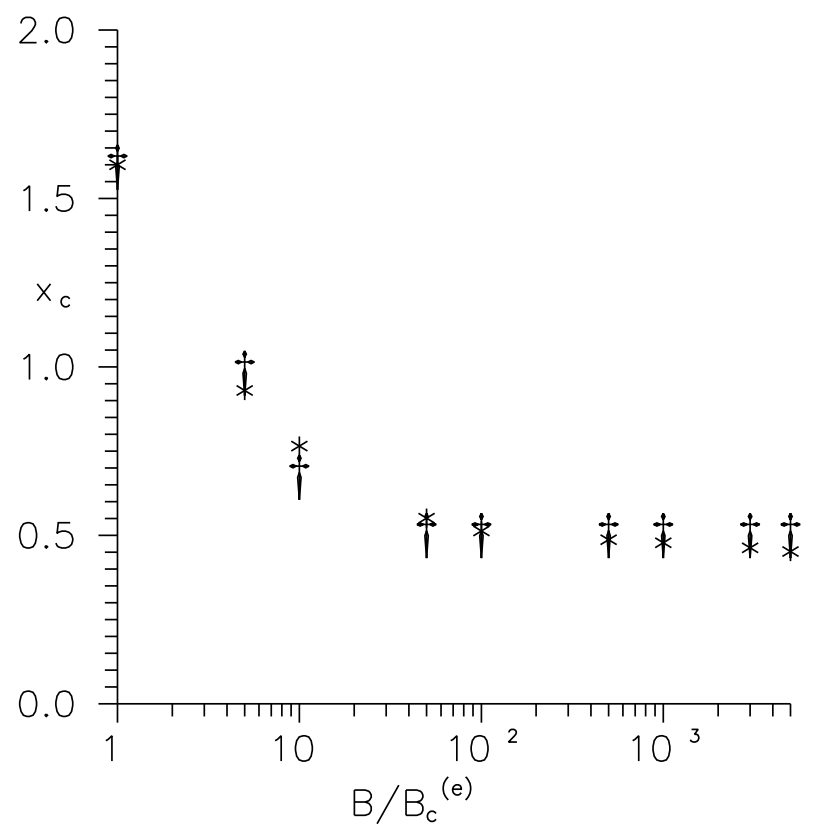

FIG. 6: The variation of critical position $\left(x_{c}\right)$ within the cell at which $E_{t o t}$ is exactly zero with the strength of magnetic field ( $*$ is the numerically evaluated points and $\dagger$ is the fitted point).

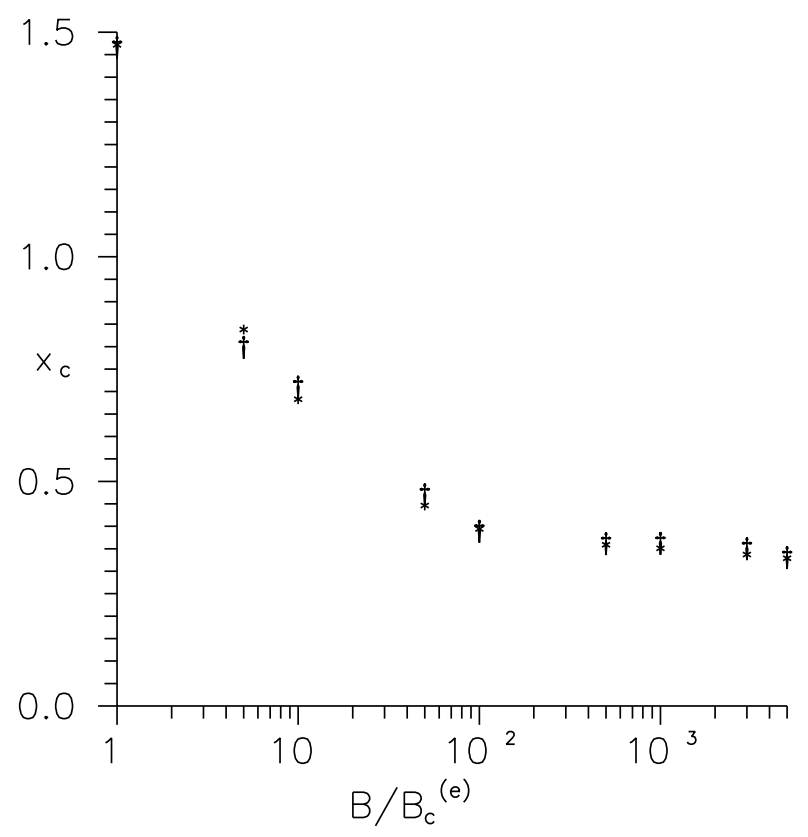

FIG. 7: The variation of critical position $\left(x_{c}\right)$ within the cell at which total pressure is exactly zero with the strength of magnetic field ( $*$ is the numerically evaluated points and $\dagger$ is the fitted point). 


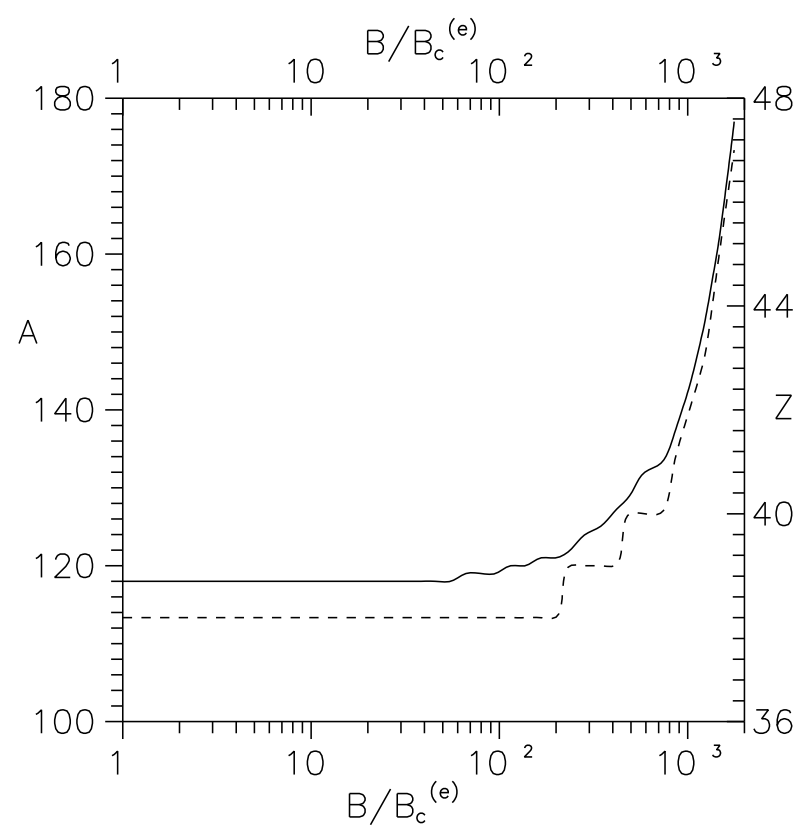

FIG. 8: Variation of critical values for $A$ and $Z$ with the strength of magnetic field $B$ (expressed as $\left.B / B_{c}^{(e)}\right)$ at which kinetic pressure becomes just zero. This result based on HW equation of state

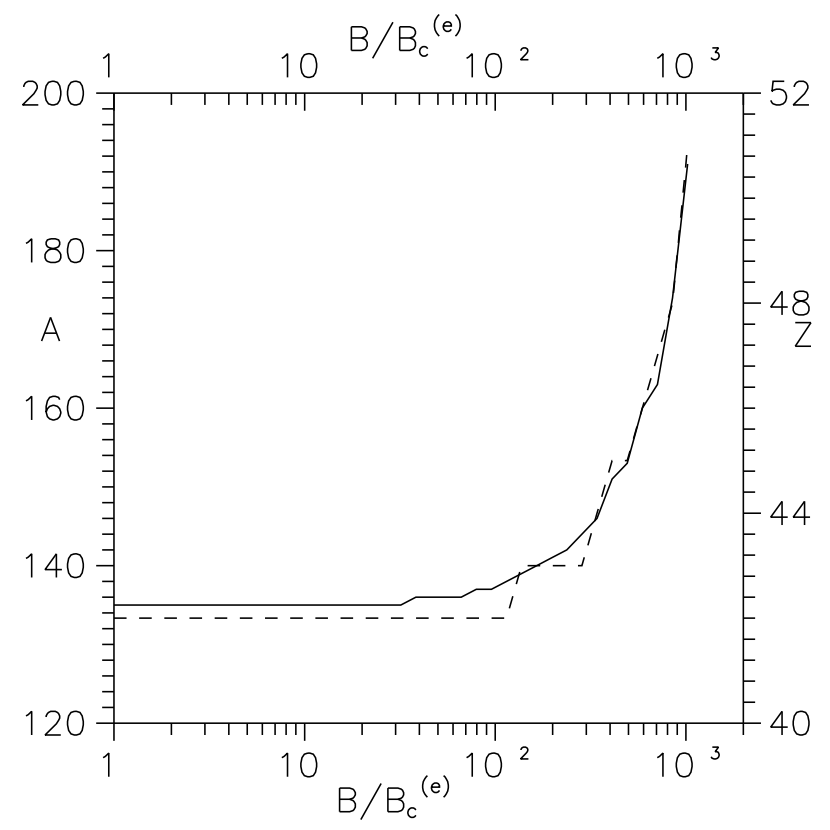

FIG. 9: Variation of critical values for $A$ and $Z$ with the strength of magnetic field $B$ (expressed as $\left.B / B_{c}^{(e)}\right)$ at which kinetic pressure becomes just zero. This result based on BBP equation of state 


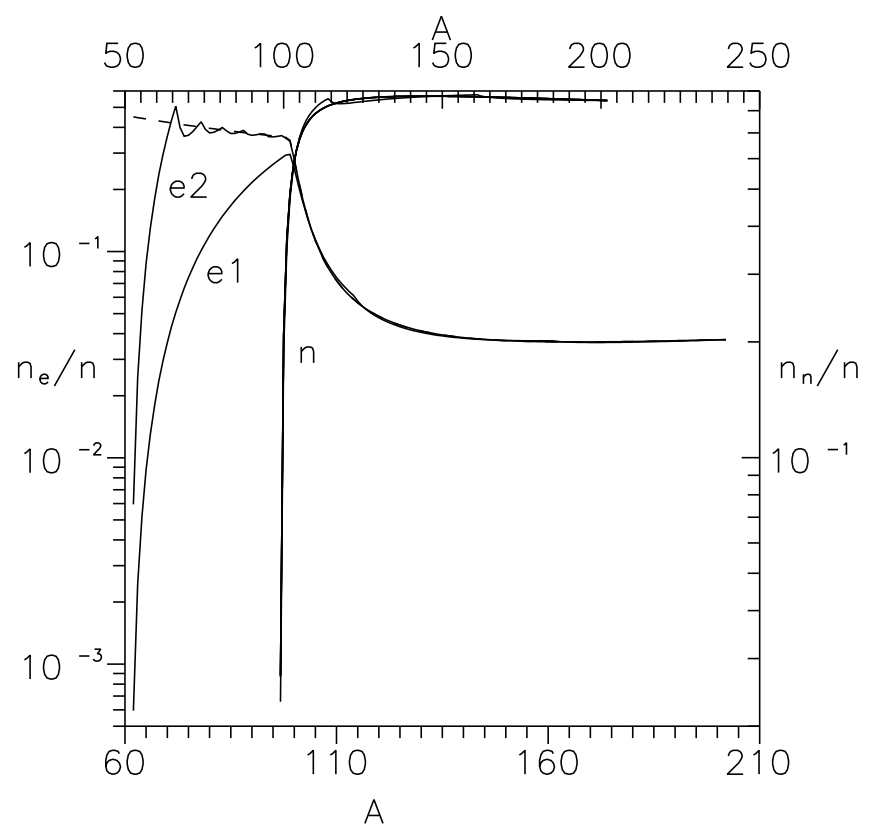

FIG. 10: Variation of the ratio $n_{e} / n$ and $n_{n} / n$ with the mass number using HW equation of state. Here $n_{e}$ is the electron density, $n_{n}$ is the free neutron density and $n=n_{n}+n_{e} A / Z$ is the total baryon density of inner crust matter. Dashed curve is for $B=0$, middle and the lower curves are for $B=10^{2} B_{c}^{(e)}$ and $10^{3} B_{c}^{(e)}$ indicated by $e 1$ and $e 2$ respectively. The curve indicated by $n$ is for $n_{n} / n$. 


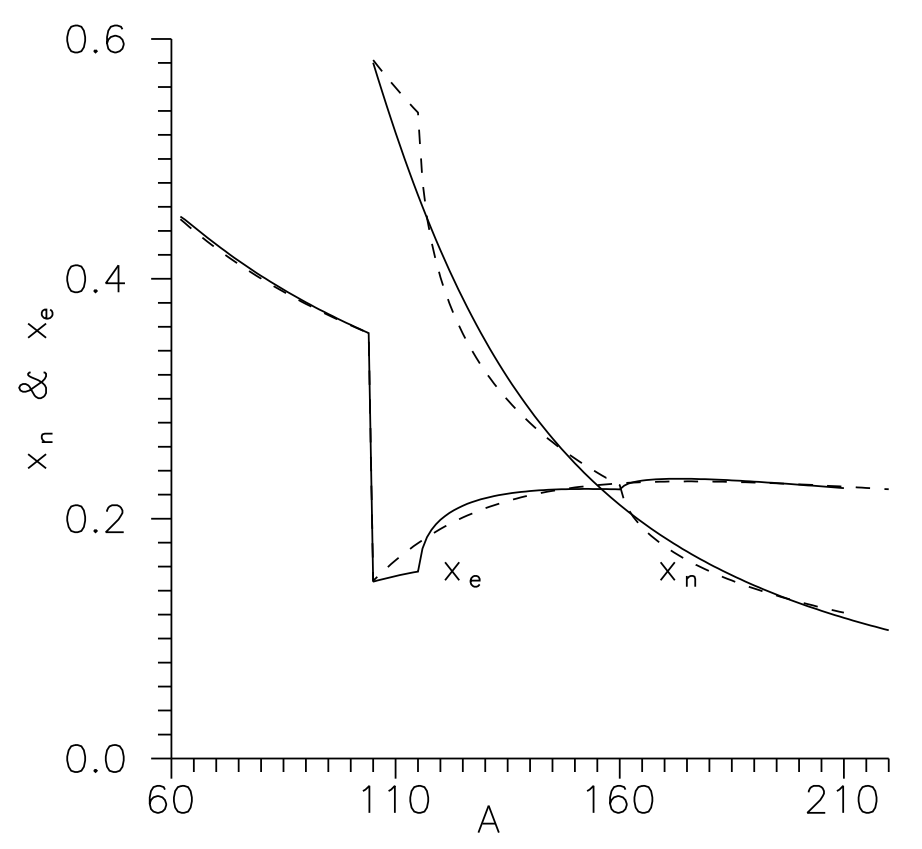

FIG. 11: Variation of the ratio $x_{e}=n_{e} / n$ and $x_{n}=n_{n} / n$ with the mass number $A$ using BBP equation of state. Here $n_{e}$ is the electron density, $n_{n}$ is the free neutron density and $n=n_{n}+n_{e} A / Z$ is the total baryon density of inner crust matter. Solid curve is for $B=0$ and the dashed curve is for $10^{3} \times B_{c}^{(e)}$ Electron part and the neutron part are indicated by $x_{e}$ and $x_{n}$ respectively.

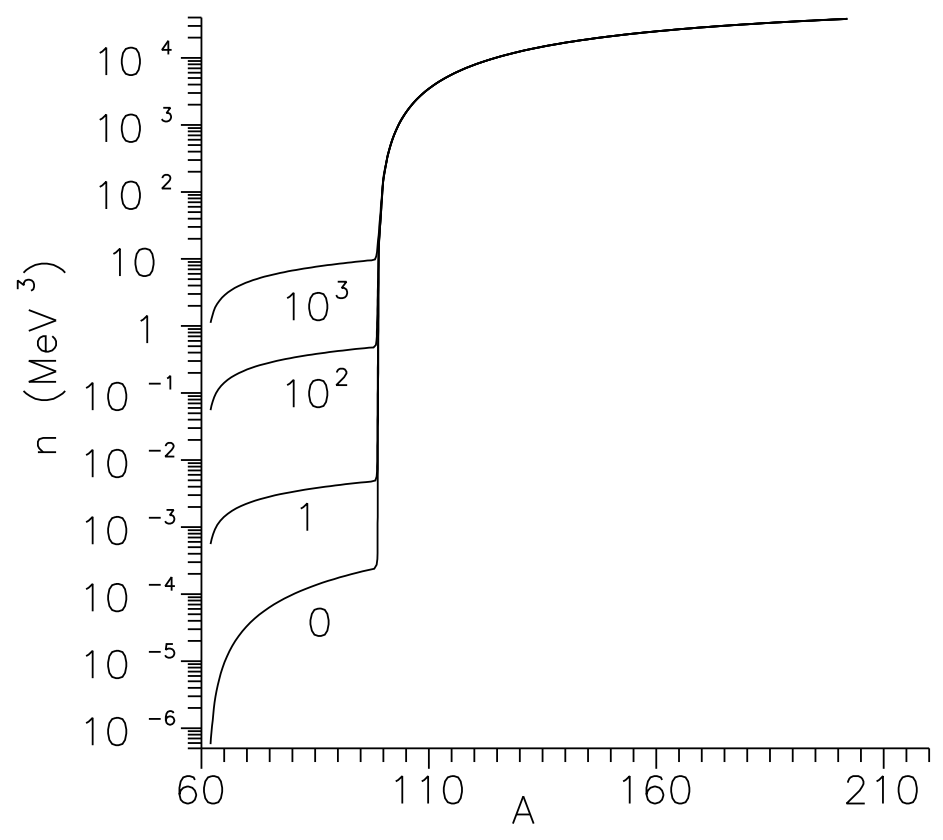

FIG. 12: Variation of total baryon density with the mass number of the nuclei, using HW equation of state for four different magnetic field strengths: $B=0, B=10 B_{c}^{(e)}, B=10^{2} B_{c}^{(e)}$ and $B=$ $10^{3} B_{c}^{(e)}$, indicated by $0,1,10^{2}$ and $10^{3}$ respectively. 


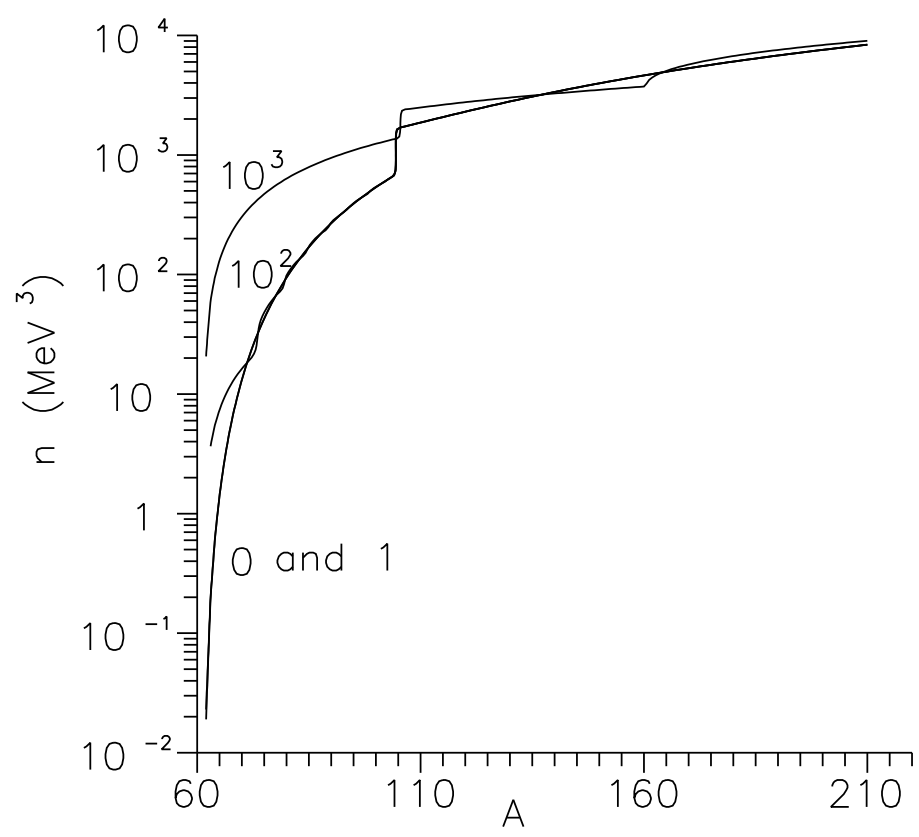

FIG. 13: Same kind of variation as in fig.12 but using BBP equation of state.

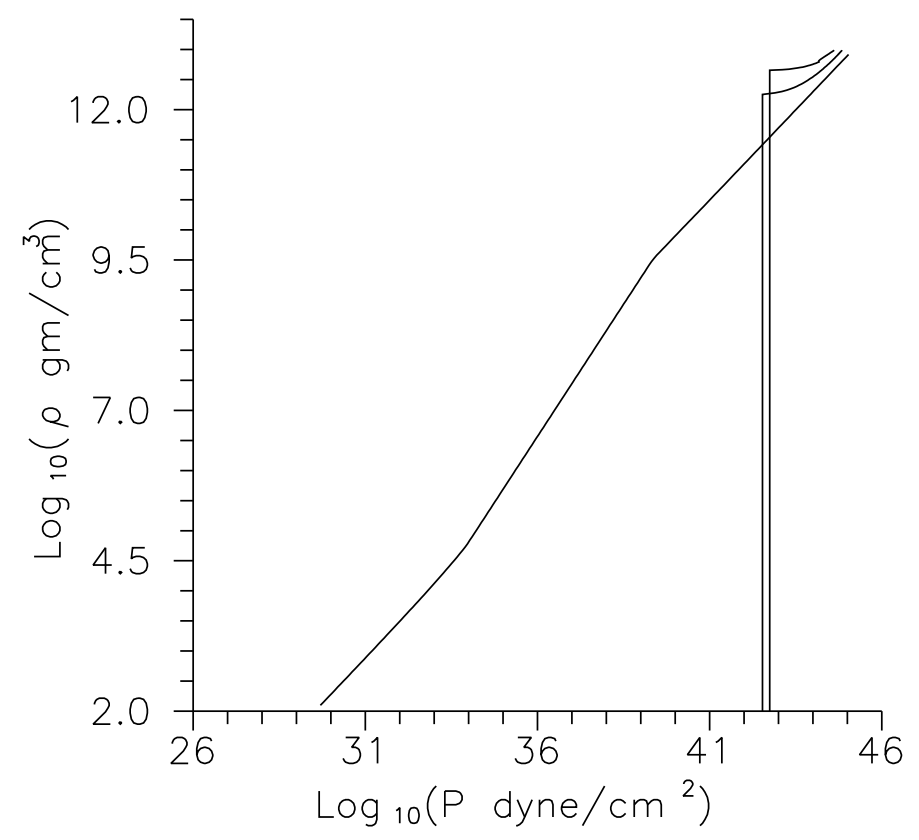

FIG. 14: Equation of state from HW mass formula. Lowe curve is for $B=0$, middle and upper curves are for $B=B_{c}^{(e)}$ and $B=10^{3} B_{c}^{(e)}$ respectively. 


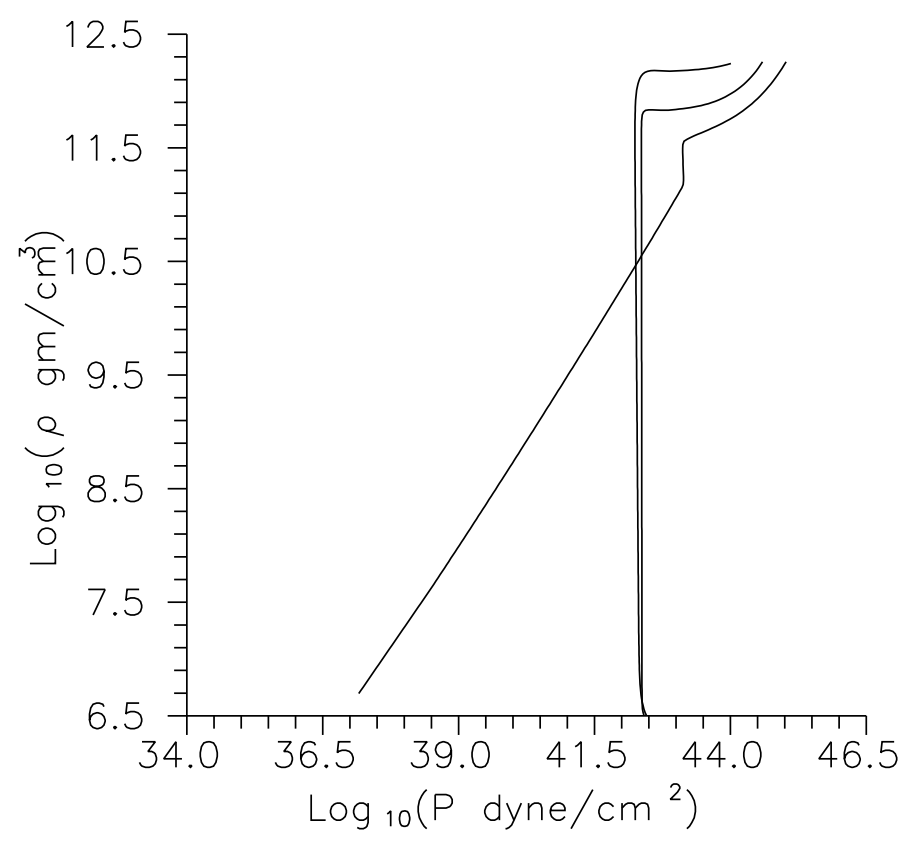

FIG. 15: Same kind of plot as shown in fig.14 but for BBP equation of state 